\title{
Abhandlung
}

Roman Kaiser*

\section{Strafrechtliche Aufgabenzuweisungen an die Bundesregierung}

DOI 10.1515/juru-2016-0118

\section{Schmähgedicht und Ermächtigung}

Der Fall Böhmermann ${ }^{1}$ hat nicht nur die Frage nach dem Verhältnis der von Meinungs- und Kunstfreiheit geschütz-

1 Einen Überblick bietet Wikipedia (de.wikipedia.org/wiki/Böhmermann-Affäre).

*Kontaktperson: Roman Kaiser, der Autor ist wissenschaftlicher Mitarbeiter am Lehrstuhl für Öffentliches Recht, Medizinrecht und Rechtsphilosophie (Prof. Dr. Josef Franz Lindner) an der Universität Augsburg. ten Satire zum Schutz der persönlichen Ehre neu aufgeworfen, ${ }^{2}$ sondern auch zwei bis dato wenig beachtete strafrechtliche Vorschriften ins Blickfeld der (nicht nur juristischen) Öffentlichkeit gerückt: § 103 StGB erklärt die »Beleidigung von Organen und Vertretern ausländischer Staaten« für strafbar, § 104a StGB stellt vier »Voraussetzungen der Strafverfolgung " auf. Das besonders im Zentrum der öffentlichen Aufmerksamkeit ${ }^{3}$ stehende Erfordernis einer Ermächtigung zur Strafverfolgung durch die Bundesregierung gibt Anlass, sich näher mit strafrechtlichen Aufgabenzuweisungen an die Bundesregierung zu

2 Zur Frage der Strafbarkeit als Beleidigung siehe Christoph, JuS 2016, 599; Fahl, NStZ 2016, 313; Rusch/Becker, AfP 2016, 201 (zudem mit rundfunkrechtlicher Bewertung).

3 Siehe etwa FAZ v. 16. 4. 2016, S. 1 u. 2; FAS v. 17. 4. 2016, S. 49; FAZ v. 22. 4. 2016, S. 1; FAZ v. 25. 4. 2016, S. 11. 
befassen. Dabei lässt sich die Ermächtigungsvoraussetzung zunächst in den größeren Rahmen strafrechtlicher Verweisungen auf das Staatsorganisationsrecht einordnen (II). Die Aufgabenzuweisungen werfen sodann und vor allem die Frage nach der konkret zuständigen Stelle auf, d.h. ob für die Ermächtigung zur Strafverfolgung die Bundesregierung als Kollegialorgan oder ein Bundesminister alleine zuständig ist (III). Schließlich ist zu zeigen, dass im Fall Böhmermann kein wirksamer Beschluss der Bundesregierung über die Ermächtigung getroffen wurde (IV).

\section{Verweisungen des Strafrechts auf das Staatsorganisations- recht}

Das StGB verweist an verschiedenen Stellen auf Institutionen des Staatsorganisationsrechts, etwa in bestimmten Straftatbeständen wie $\S \S 90,90$ b, 105 oder 106 StGB. Die im Rahmen dieses Beitrags allein interessierenden Aufgabenzuweisungen betreffen durchweg die für die Verfolgung bestimmter Straftaten aufgestellte Voraussetzung einer Ermächtigung durch politische Stellen. Die insoweit einzige Ausnahme bildet $\S 77$ a Abs. 4 StGB, der bei von einem Mitglied der Bundes- oder einer Landesregierung begangenen Antragsdelikten die jeweilige Regierung für antragsberechtigt erklärt.

Das Erfordernis einer Ermächtigung zur Strafverfolgung durch die Bundesregierung findet sich in $\S \S 97$ Abs. 3, 104a und 353a Abs. 2 StGB, durch das Bundesministerium der Justiz und für Verbraucherschutz in $\S \S 89$ a Abs. 4, 89b Abs. 4, 89c Abs. 4 und 129b Abs. 1 S. 2-5 StGB sowie durch den Bundespräsidenten in $\S 90$ Abs. 4 StGB. Strafverfolgungsermächtigungen durch die oberste Bundes- bzw. Landesbehörde sieht $\S 353$ b Abs. 4 Nr. 2 und 3 StGB vor. Weiterhin erfordern $\S 90$ b Abs. 2 StGB die Ermächtigung des »betroffenen Verfassungsorgans oder Mitglieds«, § 194 Abs. 4 StGB der »betroffenen Körperschaft« und § 353b Abs. 4 Nr. 1 StGB des »Präsidenten des Gesetzgebungsorgans«. Schließlich knüpfen $\S \S 257$ Abs. 4 und 323a Abs. 3 StGB an diese Ermächtigungserfordernisse an. Zuletzt ist $\S 77$ e StGB zu erwähnen, der für die Ermächtigungen zur Strafverfolgung $\S \S 77$ und $77 \mathrm{~d}$ StGB für entsprechend anwendbar erklärt.

\section{Zuständigkeit der Bundesregierung für die Ermächtigung zur Strafverfolgung}

Scheinen die oben (II) genannten Paragraphen die Zuständigkeit für die Ermächtigung zur Strafverfolgung jeweils entweder einem Kollegialorgan (Bundesregierung, Verfassungsorgan, Kollegialorgan einer Körperschaft) oder einem einzelnen Amtsträger (Bundesjustizminister, Bundespräsident, Präsident eines Gesetzgebungsorgans, Mitglied eines Verfassungsorgans) zuzuweisen, so stellt sich nach einem Blick in die Kommentare zum StGB (sogleich 1) die Frage, ob für die Entscheidung über die Ermächtigung in den Fällen der §§ 97 Abs. 3, 104a und 353a Abs. 2 StGB nicht die im Gesetzestext genannte Bundesregierung (als Kollegialorgan), sondern der jeweilige Fachminister zuständig ist. Eine solche ministerielle Kompetenz lässt sich indes, anders als im strafrechtlichen Schrifttum behauptet, nicht dem Ressortprinzip des Art. 65 S. 2 GG entnehmen (2). Vielmehr zeigt die Auslegung jener Normen (3), dass über die Ermächtigung das gesamte Kabinett zu entscheiden hat, während den Ministern nur die Vorbereitung und die Bekanntgabe gegenüber der Staatsanwaltschaft überlassen bleibt (4).

\section{Kommentare zum StGB}

Die Kommentare zum StGB sind sich im Grundsatz einig, dass für die Ermächtigung zur Strafverfolgung nicht die Bundesregierung als Kollegialorgan, sondern der jeweilige Fachminister zuständig ist, d.h. bei $\S 104 a^{4}$ und $\S 353 a$ Abs. $2^{5}$ StGB der Außenminister, bei $\S 97$ Abs. 3 StGB $^{6}$ der

4 AK-StGB-Anders/Mavany, Rn. 5; LK-Bauer/Gmel, Rn. 6; S/S/Eser, Rn. 3; MüKo-StGB-Kreß, Rn. 25; BeckOK-StGB-Valerius, Rn. 6; SSWVogler, Rn. 6; NK-StGB-Wohlers/Kargl, Rn. 8; SK-StGB-Wolter/Rudolphi, Rn. 8; keine Aussage bei Lackner/Kühl-Kühl und Matt/Renzikowski-Kuhli; Verweis auf $\S 97$ bei Fischer, Rn. 5; vgl. auch Rusch/ Becker, AfP 2016, 201, 202, die sich für den Fall Böhmermann auf diese allgemeine Auffassung beziehen.

5 SSW-Bosch, Rn. 4; NK-StGB-Kuhlen, Rn. 6; S/S-Perron, Rn. 6; Matt/ Renzikowski-Sinner, Rn. 6; AK-StGB-Tsambikakis, Rn. 15; LK-Vormbaum, Rn. 5; MüKo-StGB-Voßen, Rn. 24; keine Aussage bei Lackner/ Kühl-Heger, BeckOK-StGB-Heuchemer und SK-StGB-Hoyer; Verweis auf $\S 97$ bei Fischer, Rn. 5.

6 AK-StGB-Anders/Mavany, Rn. 15; Matt/Renzikowski-Dannenfeldt, Rn. 5; BeckOK-StGB-Ellbogen, Rn. 8; Fischer, Rn. 5; MüKo-StGB-Lampe/Hegmann, Rn. 22; NK-StGB-Paeffgen, Rn. 21; SK-StGB-Rudolphi/ 
Bundesminister, zu dessen Geschäftsbereich das preisgegebene Staatsgeheimnis gehört. Dabei fällt dreierlei auf: Erstens soll als Begründung - sofern eine geboten wird das Ressortprinzip des Art. 65 S. 2 GG dienen. ${ }^{7}$ Als Nachweis wird im Wesentlichen - neben Verweisungen auf andere Kommentare, eine Monographie ${ }^{8}$ und zwei weitere Aufsätze ${ }^{9}$ - ein 50 Jahre alter Aufsatz von Franz Schlichter ${ }^{10}$ angegeben, der sich als einziger näher mit der Zuständigkeit für die Ermächtigung auseinandersetzt. Zweitens wird (erstaunlicherweise nur bei § 104a StGB) in manchen Kommentaren ergänzend zur grundsätzlichen Zuständigkeit des (Außen-)Ministers noch die Behauptung aufgestellt, die Bundesregierung könne die Sache im Einzelfall an sich ziehen. ${ }^{11}$ Worauf sich diese Kompetenz gründen soll, bleibt indes gänzlich ohne Begründung.

Fast ebenso wenig klar ist - drittens -, was die Zuständigkeit des Ressortministers genau umfassen soll. So wird behauptet, der Minister vertrete »die Bundesregierung, in deren Namen er ausdrücklich oder stillschweigend handelt, nach außen «, ${ }^{12}$ seine »Erklärung gegenüber der Strafverfolgungsbehörde gilt als solche der Bundesregierung . $^{13}$ Damit könnte gemeint sein, dass sich seine Zuständigkeit darauf beschränkt, die Ermächtigung der Staatsanwaltschaft gegenüber bekanntzugeben, mithin zur Umsetzung eines Kabinettsbeschlusses nach außen tätig zu werden. Das wäre auch mit dem Begriff »Erteilung « $^{14}$ vereinbar. Gleichzeitig sei der Minister »von anderen Ressorts und dem Kabinett unabhängig « und bedürfe »keiner vorherigen Billigung durch andere Minister «. ${ }^{15}$ Dies deutet eher auf ein Selbstentscheidungsrecht hin,

Pasedach/Wolter, Rn. 13; LK-Schmidt, Rn. 14; keine Aussage bei Lackner/Kühl-Kühl, S/S-Sternberg-Lieben und SSW-Vogler.

7 »Art. 65 GG«: LK-Bauer/Gmel, § 104a Rn. 6 (dort Fn. 8); BeckOKStGB-Ellbogen, § 97 Rn. 8; NK-StGB-Paeffgen, § 97 Rn. 21; LK-Schmidt, $\S 97$ Rn. 14; MüKo-StGB-Voßen, § 353a Rn. 24; »Art. 65 S. 2 GG«: SSWBosch, § 353a Rn. 4; NK-StGB-Kuhlen, § 353a Rn. 6; S/S-Perron, § 353a Rn. 6; Matt/Renzikowski-Sinner, § 353a Rn. 6; AK-StGB-Tsambikakis, $\S 353 \mathrm{a}$ Rn. 15; »Ressortzuständigkeit«: MüKo-StGB-Kreß, §104a Rn. 25; SSW-Vogler, § 104 a Rn. 6.

8 Ringwald, Der >Arnim-Paragraf` (§ 353a StGB) und der Schutz auswärtiger Interessen der Bundesrepublik Deutschland, 2010, S. $123 \mathrm{f}$.

9 Heinrich, ZStW 110 (1998), 327, 345 (für § 353a Abs. 2 StGB); Krauth/ Kurfess/Wulf, JZ 1968, 609, 611 (für § 97 Abs. 3 StGB).

10 GA 1966, 353.

11 LK-Bauer/Gmel, § 104a Rn. 6; S/S-Eser, § 104a Rn. 3; SSW-Vogler, $\S 104$ a Rn. 6; NK-StGB-Wohlers/Kargl, § 104a Rn. 8; SK-StGB-Wolter/ Rudolphi, § 104 a Rn. 8.

12 MüKo-StGB-Lampe/Hegmann, § 97 Rn. 22; ebenso AK-StGB-Anders/Mavany, § 97 Rn. 15; NK-StGB-Paeffgen, § 97 Rn. 21.

13 LK-Schmidt, § 97 Rn. 14.

14 Etwa bei NK-StGB-Kuhlen, § 353a Rn. 6.

15 MüKo-StGB-Lampe/Hegmann, § 97 Rn. 22; ebenso LK-Schmidt, $\S 97$ Rn. 14. also eine Zuständigkeit auch für die Entscheidung über das Ob der Ermächtigung. Warum soll aber ein unabhängig und im Rahmen seiner Ressortkompetenz "selbständig (Art. 65 S. 2 GG) handelnder Minister die Bundesregierung vertreten, statt schlicht eine Erklärung in eigenem Namen abzugeben ${ }^{16}$ Hier erscheint vieles fraglich. Alle drei Umstände fordern so eine Hinterfragung der angeblichen Ministerialzuständigkeit geradezu heraus.

\section{Ressortprinzip}

Schlichter unterscheidet bei seiner Behandlung des § 100c Abs. 2 S. 2 StGB a.F. (des Vorgängers des heutigen $\S 97$ Abs. $3 \mathrm{StGB})^{17}$ anders als die StGB-Kommentare, die sich auf ihn berufen, klar zwischen der Erklärung der Ermächtigung gegenüber der Strafverfolgungsbehörde einerseits und der Sachentscheidung über die Ermächtigung andererseits. Für Erstere sei auf jeden Fall der Ressortminister zuständig, da er in seinem Geschäftsbereich die Bundesregierung vertrete. ${ }^{18}$ Dies gelte auch dann, wenn die innere Ordnung der Bundesregierung eine Entscheidung in der Sache durch das Kabinett vorsehe. ${ }^{19}$ Indes gehe das GG in Art. 65 S. 2 vom Grundsatz der selbstständigen und eigenverantwortlichen Geschäftsführung der Fachminister aus, während dem Kollegialorgan Bundesregierung nur die Entscheidung über »besonders wichtige Aufgaben, die seigentlichen Regierungsaufgaben « " vorbehalten sei. ${ }^{20}$ Wenn ein Gesetz von »Bundesregierung « spricht, müsse darunter grundsätzlich der jeweilige Ressortminister verstanden werden. ${ }^{21}$

Diese Annahme lässt sich jedoch verfassungsrechtlich nicht begründen. Die Kompetenzen der Bundesregierung werden von Art. 65 GG nicht abschließend auf Kabinett, Kanzler und Minister verteilt. ${ }^{22}$ Vielmehr gehen verfassungs- und einfachrechtliche Aufgabenzuweisungen an diese Teilorgane dem allgemeinen Art. 65 GG vor. ${ }^{23}$ Dem Gesetzgeber steht es offen, eine Kompetenz der Bundesregierung als Kollegialorgan zuzuweisen, sofern es sich

16 Dazu, dass Bundesminister bei der Erfüllung ihnen eigens zugewiesener Aufgaben nicht namens der Bundesregierung handeln, siehe Detterbeck, in: Isensee/Kirchhof, HStR III, § 66 Rn. 4.

$17 \S 104$ a StGB erwähnt Schlichter, GA 1966, 353, nur auf der ersten Seite, später hingegen nicht mehr. § 353a Abs. 2 StGB findet gar keine Berücksichtigung.

18 Schlichter, GA 1966, 353, 366.

19 Ebd.

20 Schlichter, GA 1966, 353, 367.

21 Ebd.

22 Oldiges, in: Sachs, GG, Art. 62 Rn. 9 f.

23 Hermes, in: Dreier, GG, Art. 65 Rn. 13. 
nicht um eine Aufgabe handelt, die nur im Rahmen eines Ressorts (z.B. wegen des Erfordernisses eines Verwaltungsunterbaus) erfüllt werden kann. ${ }^{24}$ Spricht eine Zuweisungsnorm die »Bundesregierung « an, so beurteilt sich die Zuständigkeit nicht nach Art. $65 \mathrm{GG},{ }^{25}$ sondern es ist durch Gesetzesauslegung zu ermitteln, ob das Kabinett, der jeweilige Ressortminister oder der Bundeskanzler gemeint ist. ${ }^{26}$ Das Ressortprinzip des Art. 65 Abs. 2 GG begründet dabei genauso wenig eine Sachentscheidungskompetenz eines einzelnen Ministers wie eine Regelvermutung besteht, dass der einfache Gesetzgeber unter »Bundesregierung " den jeweiligen Fachminister verstanden wissen will. ${ }^{27} \mathrm{Im}$ Gegenteil folgt aus Art. 62 GG die widerlegliche Vermutung, dass mit jener Bezeichnung das Kollegialorgan gemeint ist. ${ }^{28}$ Eine Zuständigkeit des Ministers lässt sich mithin nicht durch pauschalen Verweis auf das Ressortprinzip des Art. 65 S. 2 GG begründen. Vielmehr ist die zuständige Stelle mittels Auslegung der Aufgabenzuweisungsnorm zu ermitteln; sie ergibt sich nicht aus dem Verfassungsrecht, sondern aus dem einfachen Strafrecht.

\section{Auslegung der Aufgabenzuweisungen}

\section{a) "Bundesregierung"}

Der Wortlaut der $\S \S 97$ Abs. 3, 104a und 353 Abs. 2 StGB (»Bundesregierung «) legt zunächst eine Zuständigkeit des Kollegialorgans, nicht eines Ministers nahe. Im Ausgangspunkt ist davon auszugehen, dass ein Normgeber einen Begriff in dessen üblicher Verwendung in der Alltags- oder Rechtssprache gebraucht. ${ }^{29}$ Die genannten Normen des StGB verwenden einen Begriff des Staatsorganisationsrechts. Insoweit enthält Art. 62 GG eine Vermutungsregel, dass das Wort "Bundesregierung " sowohl innerhalb des GG als auch in einfachen Gesetzen das aus Bundeskanzler und Bundesministern bestehende Kollegium bezeichnet. ${ }^{30}$ Dass jedenfalls nicht stets »der jeweilige Fachminister" gemeint sein kann, zeigt etwa Art. 80 Abs. 1 GG, der bei der Bestimmung der möglichen Adressaten einer Verord-

24 Hermes, in: Dreier, GG, Art. 65 Rn. 34; Oldiges, in: Sachs, GG, Art. 65 Rn. 29.

25 Hermes, in: Dreier, GG, Art. 65 Rn. 13.

26 Oldiges, in: Sachs, GG, Art. 62 Rn. 11.

27 Oldiges, in: Sachs, GG, Art. 65 Rn. 7.

28 Detterbeck, in: Isensee/Kirchhof, HStR III, § 66 Rn. 11; Oldiges, in: Sachs, GG, Art. 62 Rn. 11a; Schröder, in: v. Mangoldt/Klein/Starck, GG, Art. 62 Rn. 14.

29 Lindner, RphZ 2016, 44, 57.

30 Nachweise in Fn. 28. nungsermächtigung expressis verbis zwischen Bundesregierung und Bundesminister differenziert. Auch das BVerfG hat festgehalten: »Im allgemeinen Sprachgebrauch bedeutet `Bundesregierung ` oder `Landesregierung ` heute im Zweifel das kollegial gebildete Verfassungsorgan «. ${ }^{31} \mathrm{In}$ dubio pro collegio. ${ }^{32}$

\section{b) Systematische Differenzierung}

Bei der Betrachtung der Systematik des StGB selbst fällt auf: Der Gesetzgeber des StGB hat die Aufgabe, über die Ermächtigung zur Strafverfolgung zu entscheiden, in den Fällen der §§ 89a Abs. 4, 89b Abs. 4, 89c Abs. 4 und 129b Abs. 1 S. 2-5 StGB jeweils dem Bundesministerium der Justiz und für Verbraucherschutz, also explizit einem Bundesminister zugewiesen. Auch $\S 353$ b Abs. 4 Nr. 2 StGB, der eine Ermächtigung durch die oberste Bundesbehörde erfordert, adressiert damit in erster Linie die Bundesministerien. Es kann somit keinesfalls behauptet werden, das Gesetz verwende stets, wenn ein Minister zuständig sein soll, das Wort »Bundesregierung « in der Bedeutung "Minister des betroffenen Ressorts «. Aus staatsorganisationsrechtlicher Sicht ist dazu - als Einschub - anzumerken: Der Gesetzgeber hat grundsätzlich zwei Möglichkeiten, eine Kompetenz einem Minister zuzuweisen. Zum einen kann er die genaue Bezeichnung eines bestimmten Ministeriums wählen, wobei ein Konflikt mit der Organisationsgewalt des Bundeskanzlers dadurch vermieden wird, dass der Wortlaut der Zuweisungsnorm aufgrund des Zuständigkeitsanpassungsgesetzes durch Rechtsverordnung geändert werden kann. ${ }^{33}$ Zum anderen kann der Gesetzgeber, wenn in verschiedenen Situationen je ein anderes Ressort betroffen ist, eine abstraktere Formulierung wie »der zuständige Bundesminister« wählen, was er etwa in $\S 21 \mathrm{~S} .2$ KSchG, § 18 Abs. 2 S. 1 PUAG oder § 1 Abs. 1 RiWG getan hat. Beide Optionen hat der Strafgesetzgeber umgesetzt, als er die Entscheidung über bestimmte Strafverfolgungsermächtigungen ausdrücklich dem Justizminister bzw. der jeweils zuständigen obersten Bundesbehörde und damit bewusst nicht dem Kollegialorgan Bundesregierung ${ }^{34}$ überantwortete.

Zudem ist daran zu erinnern, dass die in $\S 77$ a Abs. 4 StGB festgelegte Antragsberechtigung der Bundesregierung unstrittig eine Aufgabenzuweisung an das gesamte

31 BVerfGE 11, 77, 85; vgl. auch BVerfGE 91, 148, 165f.; 115, 118, 149. 32 So eine Marginalie bei Detterbeck, in: Isensee/Kirchhof, HStR III, $\S 66$ Rn. 11.

33 Dazu Brandner/Uwer, DÖV 1993, 107; Busse, DÖV 2003, 407. 34 Vgl. BT-Drs. 8/3067, S. 6f.; BT-Drs. 14/8893, S. 9. 
Kabinett darstellt. ${ }^{35}$ Die strafrechtliche Literatur muss folglich behaupten, dass das StGB unter »Bundesregierung " dreierlei versteht: erstens das Kollegialorgan in $\S 77$ a Abs. 4 StGB; zweitens einen bestimmten Minister (nämlich den Außenminister) in §§ 104a und 353 Abs. 2 StGB; drittens den Minister des jeweils betroffenen Geschäftsbereichs in $\S 97$ Abs. 3 StGB. Naheliegender erscheint es allerdings, von einer einheitlichen Verwendung von Begriffen innerhalb desselben Gesetzes, d.h. von einer sprachlichen Differenzierung zwischen Bundesregierung als Kollegialorgan und einzelnem Minister, auszugehen.

\section{c) Historie}

Legt die systematische Analyse die Normen des StGB als gleichzeitig Gegebenes zugrunde, so ist des Weiteren zu berücksichtigen, dass sie ungleichzeitig entstanden sind und folglich ein je unterschiedliches Begriffsverständnis des historischen Gesetzgebers möglich ist. Es muss sich gleichsam an eine synchrone auch eine diachrone Betrachtung anschließen. Dabei lässt sich aus der Entstehungsgeschichte jener Normen, die eine Strafverfolgungsermächtigung der Bundesregierung vorsehen, eine ministerielle Zuständigkeit ebenfalls nicht ableiten. Der 1953 eingeführte $\S 104$ a StGB war erst im Rechtsausschuss eingefügt worden; ${ }^{36}$ weder dessen Protokolle ${ }^{37}$ noch diejenigen der späteren Behandlung im Bundestag ${ }^{38}$ lassen erkennen, dass unter "Bundesregierung « der Außenminister zu verstehen ist. Sogar eher für eine Zuständigkeit des Kabinetts spricht, dass als Vorbild für das Ermächtigungserfordernis nicht zuletzt Art. 302 Schweiz. StGB diente, ${ }^{39}$ denn in der Schweiz ist für die Entscheidung über die Ermächtigung der dortige Bundesrat als Kollegialorgan zuständig. Allerdings ist dieses Wissen für die Auslegung des § 104a StGB letztlich wenig gewinnbringend, ist doch die Regierungsstruktur der Bundesverfassung der Schweizerischen Eidgenossenschaft (BV) deutlich stärker durch das Kollegialprinzip geprägt (vgl. Art. 177 BV), als es unter dem GG der Fall ist. ${ }^{40}$ Ebenso wenig geben die Gesetzes-

35 Statt aller S/S-Sternberg-Lieben/Bosch, § 77 a Rn. 8.

36 BT-Drs. 1/4250, 17.

37 Stenographische Niederschriften des 23. Ausschusses, 246. Sitzung v. 13. 3. 1953, S. 39 ff., und 248. Sitzung v. 26. 3. 1953, S. 2 u. 6. Für die Zurverfügungstellung dieser Dokumente danke ich Prof. Dr. Claus Kreß LL.M. (Cambridge).

38 BT-Prot. 1/265, S. 12995 u. 13015f.; BT-Prot. 1/269, S. 13266 f.

39 Dreher, JZ 1953, 421, 427.

40 Vgl. Hermes, in: Dreier, GG, Art. 65 Rn. 4. materialien zu $\S 97$ Abs. 3 StGB, ${ }^{41} \mathrm{zu}$ dessen Vorläufer $\S 100$ c Abs. 2 S. 2 StGB a.F. ${ }^{42}$ sowie zu $\S 353$ a Abs. 2 StGB $^{43}$ für die Annahme einer Kompetenz des Ressortministers her. Auch den StGB-Entwürfen von 1960 und 1962 kann ein solches Begriffsverständnis nicht entnommen werden. ${ }^{44}$

Allerdings hält der Regierungsentwurf zu § 353b StGB von 1979 fest, es sei »bisherige Auslegung und Praxis, unter >Bundesregierung ‘ [in $\S 353$ c Abs. 4 StGB a.F.] den für die Wahrung des Geheimnisses im Einzelfall zuständigen Bundesminister zu verstehen ${ }^{45}$ Dies beweist aber nicht die Richtigkeit dieser Auslegung, sondern deutet eher auf die Möglichkeit einer rechtswidrigen Staatspraxis hin. Eine solche Praxis kann auch nicht damit gerechtfertigt werden, sie habe schon vor 1949 bestanden, ${ }^{46}$ da Art. 52 WRV nach damaliger Rechtsauffassung die Wahrnehmung der Reichsregierung zugewiesener Kompetenzen durch den Ressortminister ohne Weiteres zuließ, was heute mit Art. 62 GG nicht mehr vereinbar ist. ${ }^{47}$ So ist es gerade, wie oben (2) gezeigt, eine kaum vertretbare Auslegung des Ressortprinzips in Art. 65 S. 2 GG, die der Annahme einer ministeriellen Zuständigkeit für die Strafverfolgungsermächtigungen zugrunde liegt.

\section{d) Gesamtpolitische Entscheidung}

Schließlich entspricht es auch dem Sinn und Zweck der Strafverfolgungsermächtigung, dass die Entscheidung über ihre Erteilung von der Bundesregierung als Kollegium getroffen wird. Das Erfordernis einer Ermächtigung zur Strafverfolgung dient einer politisch sinnvollen Handhabung der Strafrechtspflege $\mathrm{e}^{48}$ unter Berücksichtigung der gesamten Umstände. ${ }^{49}$ Zwar mag bei der Ermächtigung im Fall des $\S 97$ Abs. 3 StGB in erster Linie der Minister des betroffenen Ressorts in der Lage sein, abzuschätzen, welche Folgen sich aus der Preisgabe des Geheimnisses ergeben werden..$^{50}$ Ebenso werden bei der Entscheidung über

41 BT-Drs. 5/898, S. 34; BT-Drs. 5/2860, S. 20; BT-Prot. 5/177, S. 9534 .

42 BT-Drs. 1/2414, S. 7; BT-Prot. 1/158, S. 6325; BT-Prot. 1/160, S. $6476 \mathrm{ff}$.

43 BT-Drs. 1/2414, S. 10; BT-Prot. 1/158, S. 6329; BT-Prot. 1/160, S. $6476 \mathrm{ff}$.

44 BT-Drs. 3/2150, S. 535 u. 629; BT-Drs. 4/650, S. 576 u. 675.

45 BT-Drs. 8/3067, S. 6, unter Verweis auf Schlichter, GA 1966, 353, und Krauth/Kurfess/Wulf, JZ1968, 609.

46 Vgl. den Hinweis von Schlichter, GA 1966, 353, 369 (dort Fn. 91).

47 Herzog, in: Maunz/Dürig, GG, Art. 62 Rn. 6.

48 NK-StGB-Kargl, § 77e Rn. 1.

49 LK-Schmidt, § 97 Rn. 14.

50 MüKo-StGB-Lampe/Hegmann, § 97 Rn. 22. 
die Ermächtigung nach $\S 104$ a StGB die außenpolitischen Interessen Deutschlands im Zentrum stehen, ${ }^{51}$ aber bereits der von § 353a StGB unter Strafe gestellte Vertrauensbruch im auswärtigen Dienst wirft mehr als nur außenpolitische Fragen auf. Bei allen Ermächtigungen können - und werden in der Regel - auch innen- und justizpolitische Erwägungen eine Rolle spielen, wie der Fall Böhmermann demonstriert hat. Unter diesem Blickwinkel erscheint es zweckmäßig, wenn die gesamten Erwägungen im Rahmen der Kabinettsdiskussion vorgebracht und erörtert werden können und die Bundesregierung sodann eine Entscheidung in politischer Gesamtverantwortung trifft.

Auch die Differenz zu den $\S \S 89$ a Abs. 4, 89b Abs. 4, 89c Abs. 4 und 129b Abs. 1 S. 2-5 StGB, die jeweils eine Ermächtigung durch den Bundesjustizminister erfordern, lässt sich so erklären. In diesen Fällen sind nicht (inländische oder ausländische) Staatsorgane von den Taten betroffen. Die politische Dimension der Strafverfolgung ist in Folge dessen deutlich geringer ausgeprägt. Die Ermächtigung dient hier ganz wesentlich dazu, die Kriminalitätsbekämpfung strategisch steuern zu können und die Strafverfolgung auf schwerwiegende Sachverhalte zu beschränken..$^{52}$ Die Entscheidung darüber kann dem Justizminister überlassen werden. Zudem hat sich der Gesetzgeber wegen der unter Umständen, insbesondere in Haftsachen, kurzen Fristen gegen eine Befassung durch das Regierungskollegium entschieden. ${ }^{53}$ Eine solche Problematik besteht bei den Fällen der $\S \S 97,104$ a und 353a StGB nicht.

\section{Vorbereitung, Entscheidung, Erteilung}

Somit ergibt sich aus der Auslegung der Aufgabenzuweisungsnormen, dass jeweils die Bundesregierung als Kollegialorgan über die Strafverfolgungsermächtigung zu beschließen hat. Dem Kabinett kommt die volle Sachentscheidungskompetenz zu, während die Fachminister kraft Ressortkompetenz auf Vorbereitung und Durchführung der Entscheidung beschränkt sind. ${ }^{54} \mathrm{Zu}$ diesem »kabinettorientierten Wirkungskreis « ${ }^{55}$ der Bundesminister zählt also zum einen, die Entscheidung der Bundesregierung durch das Zusammenstellen der Tatsachengrundlage und der anzustellenden Erwägungen vorzubereiten, zum anderen, die Entscheidung über die Ermächtigung den zu-

51 MüKo-StGB-Kreß, § 104a Rn. 4. 52 MüKo-StGB-Schäfer, § 129b Rn. 24.

53 BT-Drs. 14/8893, S. 9.

54 Vgl. Oldiges in: Sachs, GG, Art. 65 Rn. 32.

55 Kölble, DöV 1973, 1, 4. ständigen Strafverfolgungsbehörden bekanntzugeben. Dabei verhält es sich keineswegs so, dass jeweils nur ein Minister sowohl für Vorbereitung als auch Durchführung zuständig ist. ${ }^{56}$ Was die Vorbereitung anbelangt, so sind die Minister zu beteiligen, aus deren Ressorts spezifisch zu den nötigen Überlegungen beizutragen ist, also neben dem Außenministerium (§§ 104a, 353a Abs. 2 StGB) bzw. dem vom Geheimnisverrat betroffen Ministerium (§97 Abs. 3 StGB) etwa auch Justiz- und Innenministerium. Der der Entscheidung zugrunde zu legende Sachverhalt, d.h. der Ermittlungsstand, wird richtigerweise vom Justizministerium festgestellt (vgl. Nr. 210 Abs. 2 RiStBV). Für die Durchführung, also die Erteilung der Ermächtigung gegenüber der Staatsanwaltschaft, bedarf es, anders als bei der Vorbereitung, des Tätigwerdens nur eines Ministeriums. Warum aber sollte es in das Ressort des Außenministers fallen, Kontakt zu inländischen Justizbehörden aufzunehmen? Dafür ist ebenfalls das Justizministerium die richtige Stelle. So verteilen sich die drei Phasen Vorbereitung, Entscheidung und Erteilung auf das Kabinett und die Ministerien.

\section{Ermächtigung im Fall Böhmermann}

Die Entscheidung der Bundesregierung, die Ermächtigung zur Strafverfolgung von Jan Böhmermann nach §104a StGB zu erteilen, verkündete Bundeskanzlerin Merkel in einer Presseerklärung am 15. 4. 2016. ${ }^{57}$ An der Entscheidung waren neben der Kanzlerin Außen-, Innen- und Justizminister beteiligt. Dabei bestanden unterschiedliche Auffassungen zwischen den Unionspolitikern Merkel und de Maizière einerseits sowie den SPD-Politikern Steinmeier und Maas andererseits. Aufgrund der daraus folgenden Stimmengleichheit habe die Stimme der Kanzlerin den Ausschlag gegeben, erklärten die beiden SPD-Minister später. ${ }^{58}$ Dieses Recht der Kanzlerin zum Stichentscheid ergibt sich in der Tat aus $\S 24$ Abs. 2 GO-BReg, ${ }^{59}$ sodass die Beschlussfassung diesbezüglich nicht zu beanstanden ist.

56 A.A. Schlichter, GA 1966, 353, 366.

57 FAZv. 16. 4. 2016, S. 1; SZ v. 16. 4. 2016, S. 2.

58 FAZ v. 16. 4. 2016, S. 2.

59 Diese Regelung hat im Ausgangspunkt nichts mit der Richtlinienkompetenz (Art. $65 \mathrm{~S} .1 \mathrm{GG}$ ) zu tun, sondern stellt grundsätzlich nur eine Methode dar, bei einer Stimmengleichheit zu einer Entscheidung zu kommen, ebenso wie etwa ein Losentscheid oder die Regel, dass eine Beschlussvorlage bei Stimmengleichheit als abgelehnt gilt. Allerdings lässt sich das ausschlaggebende Gewicht der Kanzlerin wohl nur mit deren, nicht zuletzt auf der Richtlinienkompetenz beru- 
Das Problem im Fall Böhmermann ist vielmehr ein anderes: Die Bundesregierung ist gemäß $\S 24$ Abs. 1 GOBReg nur beschlussfähig, wenn mindestens die Hälfte ihrer Mitglieder anwesend ist. Da die aktuelle Regierung aus 16 Mitgliedern besteht, hätten mindestens acht von ihnen an der Entscheidung über die Strafverfolgungsermächtigung teilnehmen müssen. Mithin wurde bei der Entscheidung von Merkel, Steinmeier, Maas und de Maizière das für die Beschlussfähigkeit erforderliche Quorum nicht eingehalten.$^{60}$ Was folgt aus diesem Geschäftsordnungsverstoß? Im Regierungsinnenverhältnis liegt ein rechtswidriger und unwirksamer Beschluss vor. Nach außen hin ist die Nichteinhaltung von Geschäftsordnungsvorgaben hingegen nur beachtlich, wenn zugleich gegen das Grundgesetz verstoßen wird. ${ }^{61}$ Das BVerfG fordert für die Beschlussfassung der Bundesregierung dreierlei: Information, Quorum und Majorität. ${ }^{62}$ Ohne diese Essentialia einer Kollegialentscheidung ist ein Beschluss dem Kollegialgremium nicht materiell zurechenbar. ${ }^{63}$ Dabei gehört auch »die Mitwirkung

hender, besonders herausgehobener Stellung innerhalb der Bundesregierung rechtfertigen, was wiederum zu Problemen führt, wenn im Kabinett ausnahmsweise lediglich ein Minister den Vorsitz führt (vgl. Epping, DöV 1995, 719, 721).

60 Dass "so gesehen« die Bundesregierung nicht beschlussfähig war, erkennt auch Günter Bannas in der FAZ v. 22. 4. 2016, S. 10, zieht aber in der Folge nicht die Konsequenz, dass damit kein wirksamer Beschluss vorliegt, da er davon ausgeht, Außen- und Justizminister hätten ohnehin »nach ihrer `Ressortverantwortung allein entscheiden können «.

61 Oldiges, in: Sachs, GG, Art. 65 Rn. 38.

62 BVerfGE 91, 148, 166.

63 Epping, DÖV 1995, 719, 720. einer hinreichenden Zahl von Mitgliedern der Bundesregierung [...] zu den Mindestvoraussetzungen dafür, dass ein Beschluss der Bundesregierung zugerechnet werden kann. « ${ }^{64}$ Ohne die Einhaltung eines Quorums liegt mithin von Verfassungs wegen kein Beschluss des Kollegialorgans Bundesregierung und folglich auch im Außenverhältnis kein wirksamer Rechtsakt vor. Die Ermächtigung zur Strafverfolgung von Jan Böhmermann war deshalb unwirksam.

\section{Fazit}

Das StGB sieht in $\S \S 97$ Abs. 3, 104a und 353 Abs. 2 StGB eine Ermächtigung zur Strafverfolgung durch die Bundesregierung als Prozessvoraussetzung vor. Die Auslegung dieser Normen hat ergeben, dass die Entscheidung über die Erteilung der Ermächtigung nicht, wie im strafrechtlichen Schrifttum behauptet, von einem Minister, sondern von der Bundesregierung als Kollegialorgan zu treffen ist. Nur die Vorbereitung dieser Entscheidung und ihre Bekanntgabe gegenüber den Strafverfolgungsbehörden fällt in den ministeriellen Verantwortungsbereich. Im Fall Böhmermann haben indes nur vier Kabinettsmitglieder über die Erteilung der Ermächtigung entschieden, was die Beschlussunfähigkeit der Regierung zur Folge hatte. Die Strafverfolgungsermächtigung war folglich unwirksam.

64 BVerfGE 91, 148, $166 f$. 